\title{
Centralización política vs autonomía educativa: Política educativa nacional adversus autonomías territorial, universitaria y escolar
}

\author{
Political centralization vs educational autonomy: \\ National political education adversus and varsity
}

\section{RESUMEN}

La reflexión sobre la premisa constitucional de la participación en la institucionalización de la política educativa nacional de la formación integral problematiza su administración autónoma. La preocupación deriva de la investigación La administración pública del derecho a la calidad de la educación. La metodología se desplegó como hermenéutica de la relación política pública-autonomía institucional. El resultado propone establecer criterios de participación intersectorial en la formulación, implementación y administración argumentativa autónoma de la política educativa nacional, de camino a transformar institucionalmente las prácticas pedagógicas.

\section{PALABRAS CLAVE}

Política, Autonomía, Gobernanza, Administración, Innovación.

\begin{abstract}
A reflexion of the constitutional premises of participation in the National Education Policy of comprehensive training, problematizes its administrative autonomy. The concern stems from the investigative research, from the administration of public law to quality education. Methodology was deployed as hermeneutics in the political relationship of public institutional autonomy. The results aim to establish intersectorial criteria participation in the formulation, implementation and argumentation in administrative autonomy of natura educative police on its way to transform institutional pedagogical practices.
\end{abstract}

\section{KEYWORDS}

Politics, Autonomy, Governance, Administration, Innovation.

\section{JULIÁN OSORIO VALENCIA}

Docente investigador de la Universidad Libre Seccional Pereira. Director del Grupo de Investigación Función Pública, Servidores Públicos, Centro de Investigaciones Socio-jurídicas, Facultad de Derecho. Magíster en Derecho Administrativo. Investigador principal del proyecto "La administración pública del derecho a la calidad de la educación".

josorio@unilibrepereira.edu.co 


\section{INTRODUCCIÓN}

El examen del problema de la institucionalización del principio constitucional de la centralización política y la descentralización administrativa, como tensión entre la política educativa derivada del mandamiento normativo de la formación integral y las prácticas administrativas descentralizadas desplegadas en su nombre, obtuvo como resultado el reconocimiento de la desviación estándar entre las actividades institucionales en nombre de las autonomías territorial, universitaria y escolar y los fines constitucionalizados.

El análisis del problema de ineficacia social de la descentralización administrativa de la política nacional de calidad de la educación, renombrada por los investigadores en términos de autonomía territorial, universitaria y escolar, se enfocó en la ponderación de la capacidad de institucionalización de la premisa constitucional de la participación democrática, para la administración del garantismo de la fundamentalidad del derecho a una educación de calidad, asumida como formación integral por competencias.

Su objeto de estudio partió del supuesto de la validez jurídica de la normativa educativa de las competencias, fundamentada como coherencia entre los fines de la reforma educativa de la formación integral para el libre desarrollo de la personalidad, con los constitucionales de la vida digna. En su desarrollo se abordó el análisis de la relación trilemática de la legitimidad política, validez jurídica, y eficacia social de la normatividad (educa- tiva de la formación integral por competencias) (Mejía Quintana, 2005, p. 313). Análisis trilemático desde el cual ponderaron la capacidad institucional del Estado y la sociedad municipal para administrar, en Derecho, los recursos disponibles para el garantismo del derecho individual y social fundamental a una educación de calidad.

Por su parte, el ámbito de la legitimidad política fue fundamentado, de acuerdo con el profesor Mejía Quintana, en los tres niveles explicitados por él. Primero, en el de la deliberación de la Asamblea Constituyente y en el debate parlamentario que produjo los desarrollos legislativos de la educación (Leyes 30 de 1992 y 115 de 1994). Segundo, en el de las movilizaciones sociales por la educación, que concretaron los dos Planes Decenales Nacionales de Educación (1996-2005 y 20062015), así como en la movilización social del primer Plan Decenal Municipal de Educación de Pereira (2007-2016). Y tercero, en la supuesta institucionalización argumentativa de la articulación del desarrollo del educativo territorial de Pereira con la política nacional de calidad de la educación para la formación integral, y con los planes decenales de educación nacional y municipal.

"Supuesta", por cuanto el avance participativo en este tercer nivel de legitimación política no se concretó, porque el contenido de la reforma promovido en la movilización social que produjo el segundo Plan Decenal Nacional de Educación y que estructuró el primer Plan Decenal Municipal de Educación de Pereira, en el contexto de sus necesidades y ca- 
racterísticas locales, no se hizo extensivo al contenido del Plan de Desarrollo Municipal 2008-2011. De este modo, indican los investigadores al diferirse la implementación del Plan Decenal Municipal de Educación como plan indicativo del Plan de desarrollo territorial, en el tiempo del Plan de Desarrollo Municipal de Pereira 2008-2011, queda en entredicho el desarrollo institucional de las prácticas administrativas participativas, optándose por criterios establecidos unilateralmente por el actor gubernativo.

Omisión que les permitió no solo formular la tesis del débil desarrollo institucional de las prácticas administrativas participativas sino también reconocer en esta debilidad la causalidad de la ineficacia social de la reforma, manifiesta en el débil impacto de la educación en los indicadores de desarrollo humano, social, productivo y ambiental en la entidad territorial de Pereira.

Ineficacia social de la educación que, al decir de los autores, puso de presente el problema de la calidad de la gestión administrativa, específicamente de la administración autónoma de la calidad de la educación. Variable que destacan del núcleo del derecho a la educación como el menos reflexionado, puesto que sobre los demás componentes del núcleo (disponibilidad, acceso y permanencia) no solo existen estudios, sino también más acciones de protección ciudadana y administrativas del Estado.

Desde esta traza, el presente análisis procura contribuir a la comprensión y explicación de la fenomenología de la administración autónoma de un desarrollo educativo de calidad, a fin de identificar los factores potenciadores de la transformación de las prácticas políticas y administrativas y, desde ellos, mejorar su pertinencia y relevancia con los propósitos sociales constitucionalizados que, de acuerdo con los autores, buscan posibilitar.

Se trata de un análisis de la administración descentralizada del têlos del neo-constitucionalismo colombiano de una formación social participativa y con valores democráticos, a través de la política nacional de formación integral por competencias, centrado en el problema del desarrollo de las capacidades institucionales de la entidad territorial, para administrar autónomamente la institucionalización del principio constitucional de la centralización política y la autonomía territorial, universitaria y escolar.

Es una reflexión sobre la capacidad institucional del Estado Nación y del Estado territorial en la concreción de la legitimidad política de la reforma educativa de las competencias en el tercer nivel de deliberación pública, que se propone materializar la participación institucional intersectorial en la formulación, implementación y administración argumentativa de los planes de desarrollo territorial para la garantía de los derechos fundamentales, en nuestro caso, del derecho fundamental a una educación de calidad.

En otras palabras, al ponderar las razones que a juicio de los investigadores imposibilitaron avanzar al tercer momento de la legi- 
timación política, se consideró la necesidad de profundizar el problema del tránsito de la gobernabilidad a la gobernanza de los planes de desarrollo territorial, entendida la gobernanza como inclusión auténtica y no formal (gobernabilidad) en los procesos de planeación, presupuesto y ejecución de la inversión pública de la institucionalidad intersectorial.

Autenticidad que encuentra su mayor obstáculo, como se observó, en el establecimiento de criterios de participación de manera unilateral y excluyente por el actor gubernativo. Con lo que, en el campo de la educación, y de acuerdo con el concepto de currículo definido normativamente, se desnaturaliza el modelo administrativo fundado en una planeación educativa participativa, inicialmente, para la definición de los criterios de selección de los conocimientos de las ciencias, las humanidades, las artes y los saberes ancestrales, provenidos de, o determinados por, las demandas educativas y formativas identificadas en los contextos de vida de los educandos, como en los del desarrollo social y de la productividad económica.

De este modo, la hipótesis de trabajo para superar el problema de la no profundización del tercer nivel de legitimidad política, en el escenario de las prácticas administrativas de los planes de desarrollo territoriales, es el establecimiento de criterios de participación institucional intersectorial para la formulación, implementación y administración argumentativa de la política educativa nacional de la formación integral por competencias, en el contexto de las necesidades y características locales.

El abordaje de este camino no es otro que el establecido constitucionalmente para la institucionalización del Estado colombiano como Estado educador, constitucional, social, democrático y ambiental de Derecho. Hipótesis que se coloca en perspectiva de trascender de una participación formal hacia una sustancial, esto es, hacia la eficacia social del servicio educativo municipal de Pereira, de camino a superar la cada vez más profunda crisis de los valores (violencia), de la productividad (pobreza) y del ambiente (deterioro ambiental social, cultural y físicobiótico).

\section{REFERENTE CONCEPTUAL}

\section{La filogenia del "Estado somos todos" y} la naturaleza del Estado educador constitucional, social, democrático y ambiental de Derecho

Para John Searle (1997, p. 10) el lenguaje es parcialmente constitutivo de la realidad social. Su trascendencia como hecho institucional requiere de la voluntad colectiva para concretarla en un hecho institucional. En nuestro caso, la realidad social parcialmente pre-figurada en el lenguaje jurídico educativo interpela la voluntad colectiva, para concretar el comportamiento administrativo imputable al mandamiento normativo del garantismo del derecho a la calidad de la educación. 
La realización del ejercicio del principio constitucional de la corresponsabilidad actualiza el problema de la naturaleza del Estado, como participación intersectorial en la administración descentralizada de la política nacional de calidad educativa. Este principio convoca la obligación del Estado y el deber de la sociedad y la familia para administrar el garantismo del derecho a la calidad de la educación. Corresponsabilidad que impele abocar la novísima concepción del "Estado somos todos", la cual rememora la antigua concepción de la Ciudad-Estado, asumida como la capacidad social de los ciudadanos para hallarle soluciones a los problemas de la polis. Capacidad que interpela la paideia, entendida como la formación en las virtudes del buen ciudadano y la educación en las artes y en la argumentación para la participación política.

Es en la polis donde el ciudadano podía darle libre juego a todas sus potencialidades deliberativas, de camino a realizar los ideales virtuosos que le permitieran vivir en Koimonía. En la comunión con los ideales de sus conciudadanos es donde surge la política. Vivencia del arte deliberativo con el otro diferente desde donde se dirimen la diversidad de intereses y puntos de vista diversos sobre la naturaleza de los problemas que afectan la calidad de la vivencia colectiva y se gestan las convenciones. De ahí que la política tenga su comienzo y su fin en la polis.

Esta forma directa y cercana de Estado llevó a Aristóteles (2007, p. 34) a considerar que la amistad era más importante que la justicia, pues ella une a las personas a través de una red de interacciones y compromisos basados en las virtudes de la lealtad, el honor y el respeto. Por esta razón, la sanción ciudadana más grave fue el ostracismo. Sócrates prefirió la muerte al exilio, es decir, a la exclusión de esa red tejida por los sentimientos de solidaridad.

Estas "interacciones y compromisos" que hoy Ilamamos "comunidad" (Jaeger, 1992, p. 137) están fundadas en "las virtudes del buen ciudadano". Estas virtudes, que hoy se objetivan en el núcleo de constitucionalidad y que orientan la acción política práctica, son los valores y principios que se aspira orienten las decisiones en la producción legislativa y la impartición de justicia. Es a través de los valores y principios constitucionalizados donde se funda el proceso de institucionalización de un Estado fuerte, legítimo, eficaz, actor decisivo en la construcción participativa de lo público.

Esta institucionalización pasa necesariamente por la apropiación social de la concepción del "Estado somos todos", propia de la democracia constitucional o participativa. Esta apropiación demanda el idóneo conocimiento científico de los problemas del desarrollo humano, social y productivo, a fin de comprenderlos, explicarlos e intervenirlos desde la diversidad de intereses y puntos de vista diferentes. Pluralidad que exige construir acuerdos a través de políticas públicas y sociales que guíen la acción del Estado, lo que significa que el Estado se instituye en una articulación dialógica capaz de elegir las mejores decisiones públicas. 
Es a través de una acción comunicativa ética entre la sociedad política en el poder del Estado, la sociedad civil que participa en la construcción social de lo público a través de la deliberación de las decisiones públicas, y la academia, que gesta y gestiona conocimientos para su apropiación social sobre la problemática del desarrollo humano, social y productivo, incluido lo económico, desde los cuales elegir las mejores decisiones públicas.

Esta concepción del "Estado somos todos" es gestora de sinergias de interacciones derivadas de interlocuciones fundadas en los conocimientos de las ciencias y los saberes ancestrales pensados por la academia, acción comunicativa que interpela a la educación, en su función social constitucional de la formación integral de un ciudadano participativo, capaz de deliberar y construir acuerdos con sentido de los valores democráticos. Es en el escenario de la participación deliberativa donde se gesta el trámite civilizado de la diversidad de intereses y complejidad de conflictos, con perspectiva del garantismo de los derechos individuales y sociales fundamentales.

De este modo, es claro que la concreción del concepto el "Estado somos todos" está en la base de una formación social deliberante, con sentido del interés público. Concreción mediada por la determinación del alcance de la responsabilidad misional de los actores político, social, académico y educativo. Alcances desde los cuales gestar sinérgicamente las condiciones recreadoras de la institucionalización del Estado constitucional, social, democrático y ambiental de Derecho como Estado garantista de la fundamentalidad de los derechos individuales y sociales.

Estas responsabilidades sectoriales son relativizadas misionalmente por la ley, especificando:

1. Que la sociedad política propicie reglas de juego claras, procuradoras de un clima de confianza que estimule la creación de ventajas comparativas que propendan por un entorno favorable. Tal efecto requiere incluir la participación del sector privado en las funciones públicas de supervisión y evaluación del cumplimiento de los compromisos, y de suministro eficiente de bienes y servicios públicos.

2. Que la sociedad civil favorezca el estudio, análisis, deliberación pública, coordinación y promoción de políticas públicas requeridas para orientar la intervención del Estado en la garantía de los derechos fundamentales. En este marco de la función de árbitro, en los conflictos de intereses de sus asociados el Estado asume a:

- Los gremios y empresarios como parte de la sociedad civil, responsables de un desarrollo productivo capaz de una adecuada ponderación entre la rentabilidad social-colectiva y la rentabilidad privada.

- Los trabajadores como propiciadores del aumento en la productividad, la innovación y el cambio tecnológico para el desarrollo productivo; partícipes e impulsores a su vez del diseño y reestructuración de las cadenas pro- 
ductivas, y de actividades asociativas y solidarias.

- La familia como actor y autor corresponsable de la educación de sus hijos, de su formación moral y democrática desde el seno del hogar, contribuyendo a transformarlo en ethos, en costumbre interiorizada, en cultura gestora del sentido de lo público.

- El sector académico, como socialmente responsable del desarrollo de la formación profesional, de la investigación científica y tecnológica, y la gestión del conocimiento, en búsqueda de una vinculación más estrecha y coordinada entre los sectores social y productivo de bienes económicos y culturales, a fin de contribuir al acrecentamiento del capital humano, condición en la base del desarrollo del capital social y del crecimiento del capital productivo.

- Finalmente, pero no en último lugar, asumiendo a la Escuela como actor y autor responsable de la innovación pedagógica en la formación de un ciudadano integral, productor de bienes materiales y culturales.

Este es el sentido y la razón de ser de la concepción y la función social del "Estado somos todos" en la construcción de la democracia participativa para el garantismo de los derechos fundamentales, Estado constitucional que se asuma no solo como sistema político o forma de gobierno, sino también como conjunto de valores, principios morales constitucionales de acción práctica, actitudes y comportamientos vividos cotidianamente por las sociedades política y civil, y por las comunidades académicas y educativas, ideales virtuosos a desarrollar desde una educación de calidad, camino de la equidad como igualdad de oportunidades para todos, a través de una formación integral por competencias para el libre desarrollo de la persona humana.

Desde luego, la institucionalización del Estado constitucional, social y democrático de Derecho, como Estado garantista de los derechos fundamentales, además de la premisa constitucional de la participación, interpela la función social del Derecho, como mediador de las relaciones implícitas entre los actores y autores del "Estado somos todos". Este uso de la razón jurídica se realiza a través del "principio del discurso", "acción comunicativa" que es fundamentada por la ciencia del Derecho, "disciplina multidimensional" (Alexy, 2002, p. 44), que "establece pautas y derroteros a las conductas que orientan la acción social" (Botero, 1999, p. 41).

Se interpela, en consecuencia, el sentido de la prevalencia del interés público por el derecho social fundamental a una educación para todos, pero no a cualquier tipo de educación sino a una educación de calidad, entendida en el ordenamiento constitucional y normativo educativo como educación para la formación integral por competencias, en los contextos problemáticos del desarrollo humano, social y productivo. Posibilitante de ciudadanos posibilitados para su comprensión, explicación e intervención. Desarrollo 
del derecho a la educación de calidad que, como anota la Corte Constitucional, realice materialmente el principio de la equidad, en virtud a que "en la medida en que la persona tenga igualdad de oportunidades educativas, tendrá igualdad de oportunidades en la vida para efectos de su realización como persona" (Sentencia T-309 de 1993), principio que hace que se comprenda por qué la educación para la formación integral es un asunto que nos compete a todos.

2. La naturaleza argumentativa de la administración pedagógica, como acción comunicativa de una práctica política legitimadora de la validez jurídica de la reforma educativa de la formación integral, por su eficacia social

Es a partir del Primer y Segundo Plan Decenal Nacional de Educación (1996-2005 y 2006-2015) que la sociedad y el Estado empiezan a sensibilizarse y entender el histórico error de creer que con solo construir plantas físicas, dotarlas y nombrar los maestros estaba garantizada la calidad de la educación. La prevalencia de la cobertura sobre la calidad ha sido un grave error.

La calidad de la educación no se garantiza con solo nombrar los maestros egresados de las Facultades de Educación (programas de licenciaturas) o de profesionalización universitaria, puesto que "las Facultades de Educación y las Normales no están formando a sus egresados en cómo enseñar a deducir o argumentar" (De Zubiría, 2011, p. 17). De hecho, según el Viceministerio de Educación
Superior "las fallas más recurrentes (...) están relacionadas con la planta docente; muchos de los profesores de estas Facultades no son de tiempo completo, no tienen estudios de posgrado y su producción académica e investigativa es escasa" (p. 7), a lo cual habría que agregar que la escasa investigación que realizan es disciplinar y no está asociada a sus prácticas administrativas, educativas y pedagógicas de la enseñanza disciplinar, y menos del reto de pensar la formación integral a través de programas y proyectos que intervengan los contextos de vida problemática de los educandos o de la ciudad. La planeación curricular de estas Facultades se hace por fuera de los contextos educativos. En el caso de Pereira se encontró que no hay líneas de investigación educativa y pedagógica, y si los profesores no conocen científicamente lo que acontece en las instituciones y en las aulas, ¿cómo van a construir una malla curricular que forme maestros capaces de trascender de una práctica de enseñanza disciplinar metafísica y compartimentada a la dinamización de un proceso de formación integral por competencias en los contextos de vida de los educandos?

Una evidencia en este sentido fue la pesquisa que se realizó a una muestra de Trabajos de Grado, que versan sobre problemas disciplinares, ninguno sobre la realidad pedagógica y educativa institucional y de aula, y si no hay conocimiento de la realidad pedagógica ¿cómo pueden transformarla desde los rediseños curriculares que dicen realizar? Excusarse con el facilismo de acusar a los maestros de los niveles inicial, básico y 
de educación media, porque entregan a los estudiantes que llegan a los programas de licenciatura sin las competencias básicas, ciudadanas y comunicativas, es asumir la actitud del avestruz, esconder la cabeza para no aceptar la responsabilidad que les compete de investigar esta realidad para transformarla.

Se está en una situación crítica. Las pruebas Saber Pro, 11으, 90 y 50 muestran que más de la mitad de los evaluados tienen resultados muy bajos en competencia lectora y escritora. El puntaje promedio del país es de 46 -de 100 puntos posibles-. Por su parte, PISA (Programa Internacional de Evaluación de Estudiantes) dice que el $78 \%$ de los estudiantes no llega al nivel 2 de lectura, es decir, no logran argumentar ni deducir. Es claro que aunque se ha hecho mucho en cobertura, es poco lo que se avanza en calidad. "Que solo el $5 \%$ de los colombianos $-y$ estoy siendo generoso- esté recibiendo buena educación es altamente preocupante, puesto que una educación de baja calidad achica la democracia" (De Zubiría, 2011, p. 17).

Es una realidad que demanda conocimiento, no solo para identificar los factores que inciden en la calidad, sino también los que posibilitan la responsabilización de todos en la formulación de una política de formación inicial de docentes y de actualización de los docentes en servicio, a partir de los factores vulneradores de la calidad identificados.

Esta demanda de conocimiento científico sobre las prácticas educativas y pedagógicas de todos los actores interpela la ética de la responsabilidad social con sentido de lo público, para comprender la disfunción entre las prácticas educativas y pedagógicas instaladas por la tradición, en relación con el comportamiento administrativo pedagógico autonómico imputable al mandamiento constitucional y legal de una formación integral por competencias en contexto.

En suma, se debe abordar el conocimiento del problema de la institucionalización del principio de acción práctica constitucional de la centralización política y la descentralización administrativa educativa. La comprensión y explicitación de esta fenomenología, en el contexto de las necesidades y características territoriales regionales, es condición sine qua non para dinamizar una sinergia trascendente de la gobernabilidad Estadocéntrica, de un ejecutivo nacional que se hace cada vez más proclive a la centralización administrativa, "argumentando" la incapacidad institucional de las entidades territoriales para administrar autónomamente el desarrollo educativo.

Se simula una descentralización a través de la certificación educativa a las entidades territoriales para que administren los recursos del SGPT que vienen con destinación específica al pago de nómina y del componente logístico de la calidad, sin recrear condiciones a la administración pedagógica de la calidad educativa. Se insta, además, a que incrementen los recursos propios, para atender la prestación del servicio y no el garantismo del derecho a la calidad de la educación, de 
conformidad con los mandamientos constitucionales y legales.

Este mal ejemplo es imitado por el Estadocentrismo local, quien no solo monopoliza la ejecución y asignación de los recursos propios y hasta los que se pueden del SGPT, sino que se muestra reticente a debatir lo público, ofrecer razones y rendir cuenta de sus actos, en relación, no con los resultados de sus decisiones en las inversiones, como lo hacen con despliegue publicitario sin par, sino con los impactos de la formación integral en contexto, como manda la Constitución y la ley, mal ejemplo que emulan con prestancia también en la formulación de las políticas públicas educativas territoriales.

Estos procesos "participativos" aunque consideran formalmente la opinión de la ciudadanía, no constituyen procesos auténticos de inclusión integradora de una relación interdependiente y horizontal para su formulación participativa. De hecho, los actores invitados asisten atendiendo criterios preestablecidos por la "sapiensa" del establecimiento en el poder del Estado territorial. No son criterios construidos conjuntamente para la formulación, implementación y ejecución de las políticas, planes, programas y proyectos.

Se hace necesario trascender hacia toma de decisiones que involucren a los demás actores que estructuran el Estado educador para que, en el ámbito de la calidad educativa, se destinen a procurar políticas públicas y sociales territoriales contextualizadoras de la política nacional de calidad de la educación y de formación pedagógica docente, políticas contextuales que orienten la formulación y ejecución de un currículo de formación de maestros, con criterios de todos los actores, atendiendo los criterios legales de formación pedagógica y disciplinar establecidos por el Estado para las instituciones formadoras de maestros a fin de alcanzar los logros de los estándares y lineamientos curriculares por competencias, decisiones que trasciendan la decisión Estado-céntrica de destinar los recursos para capacitación docente a criterio del gobernante de turno, amoralidad que afecta la responsabilidad constitucional de gobernantes e instituciones formadoras que ponen el énfasis en lo que saben hacer, capacitaciones para una formación disciplinar fragmentada y descontextualizada que, en lugar de trascender, persiste en la reproducción de una práctica educativa memorística, reactiva, sin capacidad de repensarse a sí misma críticamente y propositivamente en relación de pertinencia académica con las demandas del desarrollo social.

Se requiere la formulación participativa intersectorial de políticas públicas y sociales de ciencia, tecnología, educación y cultura que orienten la trascendencia, o al menos la concienciación, de la gobernanza, de una práctica administrativa pública incluyente de todos los actores y autores del hecho educativo, en el escenario de una administración argumentativa no solo del desarrollo de la prestación de un servicio educativo, sino del garantismo de la formación humanística, científica y tecnológica de ciudadanía responsable de la endogenización de su propio 
desarrollo educativo, cultural, humano, social y productivo, administración argumentativa intersectorial para la formulación de una política pública territorial de investigación, educación e innovación, tal como se propuso en el Primer Plan Departamental de Ciencia, Tecnología e Innovación, que erija el postulado constitucional de la formación integral en los contextos problemáticos del desarrollo humano, social y productivo regional, política que oriente la acción del Estado educador constitucional, social, ambiental y democrático de Derecho, para que permita la formación de una ciudadanía posibilitada para participar en la generación de opinión pública deliberante y eficaz en el ejercicio de la gobernanza de la sostenibilidad de la convivencia social y la auto-sostenibilidad productiva, gobernanza que incluya a los actores sociales, académicos y educativos en los procesos de planeación, presupuestación, programación y ejecución de la política educativa, incluso hasta de los procesos de fiscalización y rendición pública de cuentas, y de responsabilización social pública, colectiva-individual, administración argumentativa que delibere la dilucidación de la contraposición entre dos alternativas de sentido educativo correspondientes a dos cosmovisiones administrativas educativas con contenidos de formación diferentes, que plantean numerosos problemas ligados a las finalidades de la gestión, a su dimensión ideológica y a su definición misma: una, centrada en la racionalidad instrumental para la competitividad del mercado, que se mueve dentro de los parámetros administrativos de la racionalización educativa tayloriana, y otra, buscando:
"Reconstruir un sentido de la educación que se centre directamente en la condición humana y sepa desarrollar nuevas formas de humanismo, más para la cooperación antes que para la competitividad, que rescate las humanidades en su sentido más contemporáneo para la formación de ciudadanas y ciudadanos responsables, actores en la sociedad civil, comprometidos con un sentido de democracia participativa que permita buscar nuevos caminos de cooperación" (Hoyos, 2007, p. 15).

Alternativas administrativas a dilucidar desde la pregunta por el propósito de las comunidades educativas: ¿ Formar para reproducir el estado de cosas inequitativo o para transformar las cosas? ¿Formar solo para la producción de bienes económicos o para la producción de bienes espirituales y materiales?

La anterior argumentación es extensiva a la lógica de los currículos centrados en la reproducción del modelo educativo transmisionista de información y conocimientos por fuera de los contextos, o posibilitadores de nuevos modos de pensamiento y acción en contexto, gobernanza que dilucide el problema de la filosofía de la educación y la demanda de la administración de su desarrollo desde la argumentación administrativa de la relación medios-fines, deliberación pública que permita recuperar la importancia de las filosofías de la administración pública en su pretensión de interpretar los problemas administrativos, en este caso de la forma- 
ción humanística, científica y tecnológica en contexto, orientando la construcción de sus argumentos y categorizando el sentido, condiciones y responsabilidades de la administración pública educativa, filosofías administrativas de lo público educativo desbordantes de la visión factual administrativa de la racionalización educativa tayloriana, centrada en la finalidad del control a la eficiencia financiera, indicador intermedio desarticulado del indicador de impacto en los fines de la formación integral, desarticulación elusiva de la construcción de estrategias argumentativas fundadas en la reflexión de la experiencia administrativa de lo público educativo para una comprensión del sentido de una práctica administrativa de lo público con enfoque de derechos, vacío llenado "con metáforas de economía y gerencia, cuyos argumentos se apoyan únicamente en la magia de los números, para una interpretación que no problematiza el fondo social y público de la administración educativa" (Hood y Jackson, 1997, p. 35), gobernanza de la política territorial de calidad de la educación que oriente el desarrollo y fortalecimiento de la capacidad administrativa territorial para que las comunidades educativas apropien diversos discursos filosóficos de la educación, que amplíen la visión que orienta el reflexionar pedagógico de una experiencia educativa, cosmovisiones desde las cuales se problematice el acontecer educativo, y su pertinencia académica con la relevancia social, en desarrollo de los fines constitucionales y legales del Estado educador, que articule la corresponsabilidad del nivel central con el descentralizado, en la co-gestión de las condiciones para la administración argumentativa pedagógica curricular con enfoque de derechos, potestativa de las autonomías territorial, universitaria y escolar, en su función pública de garantizar el derecho social fundamental a una educación de calidad para el desarrollo humano y social, y el crecimiento productivo.

\section{La problemática de la administración autó- noma del desarrollo educativo}

Sintéticamente, se indica que la problemática de la administración autónoma del desarrollo educativo se dilucidó en términos de la débil institucionalización del principio constitucional de la participación ciudadana en la concreción de la descentralización administrativa con enfoque de derechos. Seguidamente, su efecto visible se reconoció en la artificiosa articulación de los planes territoriales de desarrollo educativo con los planes decenales nacional y local de educación, y su causalidad en la prevalencia de una práctica gubernativa y administrativa privilegiadora del interés particular de los directorios políticos por sobre los intereses públicos educativos, con lo que se aplaza en el tiempo no solo la necesaria estructuración de la política educativa nacional de estándares y lineamientos curriculares por competencias en los planes de desarrollo, sino también la necesaria articulación de los PEI al plan de desarrollo territorial.

De esta manera, los factores constitucionales de la participación y del garantismo del derecho a una educación de calidad para todos se vulneran desde la incuria del Ministerio 
de Educación para fortalecer la capacidad institucional de la entidad territorial, a través del relacionamiento idóneo de los niveles político y técnico. Desde luego, se sugiere trascender de la prédica enfática de los expertos contratados por el Ministerio para implementar sus proyectos en las regiones, hacia el conocimiento real de los saberes pre-teóricos de los servidores públicos. Es desde el conocimiento y reconstrucción de la estructura de sus saberes pre-teóricos, como precisa Habermas (2005) en el objeto de las ciencias sociales, como podrá el Ministerio dinamizar la capacidad institucional de las entidades territoriales para hacer del derecho a una educación de calidad asuntividad de todos.

DE LA GOBERNABILIDAD A LA GOBERNANZA O DE LA ADMINISTRACIÓN FORMAL A LA ADMINISTRACIÓN ARGUMENTATIVA DESCENTRALIZADA TERRITORIAL Y ESCOLAR

Es a través de la formulación de políticas públicas y sociales de educación, desde las cuales orientar la acción del Estado en sus niveles territoriales para administrar la recreación de condiciones subjetivas y objetivas al mejoramiento de la calidad de la educación, sinergia problematizadora de la gobernabilidad Estado-céntrica, esto es, de un ejercicio del Gobierno y la administración pública a través de políticas públicas gestadas exclusiva y excluyentemente por el Ejecutivo, monopolizando la ejecución y asignación de las funciones públicas del Estado, entre ellas la destinación de las inversiones, mostrándose de paso reticente a debatir su pertinencia y relevancia respecto del interés público, y a ofrecer razones y rendir cuenta de sus actos.

Desde luego, "argumentos" no faltan. Por ejemplo, que las políticas públicas educativas en marcha consideran la opinión de la ciudadanía en las Mesas de Trabajo de formulación "participativa" del Plan de Desarrollo Municipal, o que permanentemente se realizan actos de rendición pública de cuentas, desde luego que sí, pero estos actos son formalismos legales que simulan hacer lo que efectivamente no hacen.

En realidad las convocatorias para participar de la formulación de los planes territoriales de desarrollo no constituyen procesos de participación auténtica, sino conductistas.

De hecho, los criterios de participación no son concertados con fundamento en las cosmovisiones participativas de los convocados, razón por la cual no son procesos de inclusión integradora de una relación interdependiente y horizontal fundada en metodologías e instrumentos dinamizadores de conversaciones informadas e inteligentes.

Aparte de ello, las decisiones en la destinación de las inversiones las toma el Gobierno sin más criterio que el poder que ostenta. Desde luego, hay que reconocer como avance el ejercicio de los presupuestos participativos, empero estos procesos requieren de un mayor conocimiento para posibilitar que los instrumentos de la planeación y de los presupuestos participativos formales y conductistas gocen efectivamente de mayor apropiación social y mayor cobertura del ámbito 
decisional, problematización de la gobernabilidad a asumir como argumentación administrativa de un ejercicio de gobernanza, esto es, de vinculación de los demás actores estructurantes del Estado educador, como aconteció en la movilización social que estructuró el Primer Plan Decenal Municipal de Educación de Pereira, que estableció como propósito la participación de la sociedad política con la sociedad civil y el sector privado, para hacer un ejercicio de realización de la educación como un propósito de todos, tránsito de la gobernabilidad a la gobernanza ponderado en los dos planes decenales nacional y en el Primer Plan Decenal Municipal de Educación, así como en el Plan Departamental de Ciencia y Tecnología. Este último fue más allá, cuando propuso la formulación participativa de una política pública y social de educación, investigación e innovación, propuesta que, de concretarse en voluntad política de la clase dirigente local, posibilitaría orientar el ejercicio de la gobernanza de la administración pública educativa de los sistemas educativos y de ciencia, tecnología e innovación, a partir de una argumentación administrativa de la prestación de un servicio educativo garantista de la formación humanística, científica y tecnológica de ciudadanía responsable de la endogenización de su propio desarrollo educativo y cultural, y del fomento de la investigación científica por parte de la academia local y de la empresa privada.

Gobernanza que supere el esquema de gobernabilidad territorial centrado en la visión unilateral y no participativa del gobernante de turno, que posibilite el empoderamiento del poder del conocimiento sobre el poder narco y de la corrupción a través del desarrollo de una capacidad institucional y organizativa de los sistemas regionales educativo y de ciencia y tecnología, capacidad institucional política más decisiva incluso que el poder económico, práctica administrativa intersectorial de los actores políticos y gubernativos, empresariales, sociales, académicos y educativos, en los procesos de planeación, presupuestación, programación y ejecución de la política pública y social de educación, investigación e innovación, extensiva a los procesos de fiscalización y rendición pública de cuentas, y de responsabilización social pública, colectiva-individual.

Tránsito hacia una gobernanza que articule el compromiso y participación ciudadanas, académica, empresarial y gubernativa en todas y cada una de las etapas de concepción, diseño e implantación de una política pública y social territorial de educación, investigación e innovación que redefina las funciones de la administración educativa certificada y descentralizada, con la categorización e interrelación de los diferentes ciclos y niveles de la educación curricular en todo lo que hace referencia al proceso de la formación moral, tecno-científica y productiva del novel ciudadano, administración argumentativa intersectorial deliberante de la dilucidación de la contraposición entre dos alternativas de sentido educativo correspondientes a dos cosmovisiones administrativas educativas con contenidos de formación diferentes, cosmovisiones que plantean numerosos proble- 
mas ligados a las finalidades de la gestión, su dimensión ideológica y su definición misma:

a) Una, centrada en la racionalidad instrumental para la competitividad del mercado, que se mueve dentro de los parámetros administrativos de la racionalización educativa tayloriana.

b) Otra, buscando "reconstruir un sentido de la educación que se centre directamente en la condición humana y sepa desarrollar nuevas formas de humanismo, más para la cooperación antes que para la competitividad, que rescate las humanidades en su sentido más contemporáneo para la formación de ciudadanas y ciudadanos responsables, actores en la sociedad civil, comprometidos con un sentido de democracia participativa que permita buscar nuevos caminos de cooperación" (Hoyos Vásquez, 2007, p. 15).

Las anteriores alternativas administrativas se deben dilucidar desde la pregunta por el propósito de las comunidades educativas: ¿Formar para reproducir el estado de cosas inequitativo o para transformar las cosas?

Argumentación extensiva a la lógica de los currículos centrados en la reproducción del modelo educativo transmisionista de información y conocimientos por fuera de los contextos o posibilitadores de nuevos modos de pensamiento y acción en contexto, gobernanza que dilucide el problema de la filosofía de la educación y la demanda de la administración de su desarrollo desde la argumentación administrativa de la relación medios- fines, deliberación pública que permita recuperar la importancia de las filosofías de la administración de lo público educativo, filosofías desbordantes de la visión factual administrativa de la racionalización educativa tayloriana, centrada en la finalidad del control a la eficiencia financiera, sin arreglo a los fines de la formación integral, filosofías argumentativas para la construcción de estrategias fundadas en la persuasión, a través de la reflexión de la experiencia administrativa de lo público educativo, gobernanza articuladora de la corresponsabilidad del nivel central con el descentralizado, en la co-gestión de las condiciones para la administración argumentativa pedagógica curricular, legitimadora de las autonomías territorial, universitaria y escolar, en el ejercicio de su función pública de garantizar el derecho social fundamental a una educación de calidad, que pone de manifiesto la necesidad de profundizar el conocimiento de las ciencias administrativas de lo público para comprender la escisión entre el comportamiento imputable a la normativa educativa de las competencias y las prácticas institucionalizadas en su nombre.

De hecho, el actual vacío de ciencia impide des-encubrir la intencionalidad política de esta escisión, la que, dicho sea de paso, busca mantener ilegalmente la inocua tradición administrativa del transmisionismo de información y conocimientos por fuera de los contextos de vida de los educandos, prevaricación que aplaza las posibilidades de la efectiva apropiación social de los conocimientos científicos y humanísticos, y con ella la liberación de la esclavitud de la ignorancia 
(Freire, 1996, p. 23) de una sociedad anómica, proclive a la charlatanería de la pseudociencia, a la ilegalidad y a la pasividad propia de quien espera un mundo dado por el azar, por Dios, por la renta o el crimen.

En suma, el reto es por una ética orientadora, entre otras, del sentido de la cultura de la legalidad apropiado por la sociedad y, de manera particular, por las comunidades educativas en todos los niveles, las Facultades de Educación y demás programas formadores de maestros, así como por las Secretarías de Educación de las entidades territoriales y por el propio Ministerio de Educación respecto del comportamiento institucional imputable a la normativa educativa de estándares y lineamientos curriculares por competencias y su administración autónoma, ética de la legalidad extensiva al ejercicio del poder coactivo o garantista del Estado constitucional, social, democrático y ambiental de Derecho, poder no ejercido por el Ministerio de Educación para regular el ejercicio de las autonomías territorial y universitaria, y por las Secretarías de Educación para el ejercicio de "la suprema función de inspección y vigilancia" a la institucionalización de la autonomía escolar y universitaria, en relación con la administración argumentativa de la garantía de la formación integral.

Desde luego, se sabe que legalmente las Secretarías de Educación territoriales no tienen potestad jurisdiccional sobre la educación superior, pero sí sobre la política territorial de calidad y formación docente, desde don- de pueden regular la idoneidad de los programas de formación inicial y en servicio.

Sin duda, la trascendencia hacia la administración argumentativa es la alternativa de la legitimación política del Estado educador colombiano, puesto que la gobernanza y moralidad de las prácticas políticas, administrativas y educativas autónomas de las entidades territoriales y sus sistemas educativos son el camino de la validación de la normatividad educativa de las competencias, a partir del factum de los indicadores de eficacia social, reto que interpela la actividad científica administrativa de lo público, de manera particular, al derecho administrativo educativo.

\section{METOdOLOGÍA}

La investigación se desplegó con un enfoque metodológico hermenéutico socio-jurídico, el cual se orientó a contrastar la descripción fenomenológica del comportamiento administrativo imputable al principio de la corresponsabilidad del Estado, la sociedad y la familia en la administración del garantismo del derecho a una educación para la formación integral por competencias, con las prácticas administrativas instaladas a nombre del principio constitucional y legal de la autonomía administrativa territorial, universitaria y escolar. Su resultado permitió establecer que la ineficacia social del modelo administrativista formal instalado respecto de la garantía del derecho social fundamental a una educación de calidad para la formación integral se puede trascender desde una voluntad colectiva 
orientada a posibilitar el tránsito de la gobernabilidad a la gobernanza.

\section{CONCLUSIONES}

Las condiciones de institucionalización de la descentralización administrativa de la política nacional de calidad de la educación demandan comprenderse, a fin de contribuir a la concienciación de la necesidad de transitar de una administración formalista y excluyente hacia una administración argumentativa incluyente, donde se fortalezca la capacidad institucional de los actores para construir criterios de intervención, apoyándose en los conocimientos de la investigación científica.

El camino de la institucionalización o desarrollo de las autonomías territorial, universitaria y escolar es la participación argumentativa de los actores de la intersectorialidad Estado, empresa, sociedad civil, academia y comunidad educativa, en la administración pedagógica de los niveles de educación inicial, básica y media.

Si se entiende la autonomía educativa como acción comunicativa argumentativa, esta se constituye en escenario de la construcción de acuerdos, para la articulación de los planes territoriales de desarrollo educativo con los planes decenales nacional y local de educación. Así, no solo se favorece la necesaria estructuración de la política educativa nacional de estándares y lineamientos curriculares por competencias, sino también la necesaria articulación de los PEI al plan de desarrollo territorial.
De este modo, la comunidad educativa podrá asumir "el currículo como espacio de organización (argumentativa) de las partes constitutivas o interdependientes del proceso de formación integral" (Posner, 1999, p. 129), en el contexto de las necesidades y características territoriales, y "la pedagogía, como discurrir de la teoría a la práctica" (Hoyos Vásquez, 2007, p. 3). Estos son los criterios de acción práctica, desde los cuales se puede mejorar la capacidad de los estudiantes de pensar autónomamente, y argumentar y resolver problemas de forma efectiva, en sus contextos de vida.

El establecimiento de la diferencia cualitativa entre gobernabilidad Estado-céntrica excluyente y gobernanza inclusiva se puede advertir, parafraseando al doctrinante Ferrajoli (2009), en la disyuntiva entre garantismo y autoritarismo, al notar que "además de configurarse como una alternativa teórica entre modelos diversos de ordenamiento penal" (en nuestro caso, administrativo) "será consecuentemente utilizada para interpretar el conjunto de todas las oposiciones localizables dentro del mismo ordenamiento entre principios de legitimación y praxis, entre normatividad y efectividad, entre modelo constitucional y funcionamiento de las instituciones penales" (administrativas) (Ferrajoli, 2009, p. 26), principios de legitimación política por la sociedad civil derivada de la administración argumentativa de una praxis educativa asumida como acción comunicativa, fundada en el principio del discurso (Habermas, 2005, p. 13), connotante del sentido deliberante de una praxis administrativa au- 
tonomista de una función pública educativa, asociada a la correlación entre desarrollo normativo y eficacia social del comportamiento institucional educativo imputable a los fines del modelo constitucional del desarrollo humano, base de los capitales social y productivo, praxis administrativa argumentativa utilizada para interpretar el conjunto de todas las oposiciones localizables (...) entre principios de legitimación y praxis, entre normatividad y eficacia social, pero, ante todo, de la oposición entre la legitimación política de la sociedad y la del poder político del Estado, desde la dimensión del conocimiento, como razón pública societal y no como racionalidad instrumental del Estado al servicio de la globalización del mercado, administración argumentativa intersectorial que supere el amiguismo, el favoritismo y el afán de agotar el presupuesto por intermedio de contratos para acciones y programas puntuales, descontextualizados e intrascendentes, sin impacto o con mínimo impacto en los establecimientos educativos, los cuales siguen trabajando con métodos tradicionales, desconociendo la realidad de los nuevos estudiantes y por tanto con altos niveles de deserción y fracaso escolar, administración argumentativa intersectorial que ponga la escuela de frente a la realidad, acompañada por una Secretaría de Educación moderna, apoyada por un Ministerio de Educación con directrices relevantes para fortalecer la capacidad institucional autonómica territorial, universitaria y escolar, dejando en el pasado propuestas programáticas que se agotan en los programas de gobierno.
Hay que cambiar el enfoque estratégico conformando grupos de voluntarios integrados por docentes y demás agentes de la comunidad interesados en asumir procesos investigativos contextualizados con base en la realidad de sus comunidades, tendientes a formar a sus estudiantes de acuerdo con sus necesidades, buscando que la educación sea pertinente al entorno y por tanto a procesos educativos basados en competencias específicas para solucionar problemas reales y concretos sentidos por esas comunidades educativas, lo cual demanda de la Administración Municipal desarrollar procesos de actualización y formación de docentes centrados en las competencias requeridas, tal como se estableció en el Plan Territorial de formación de directivos docentes y docentes en servicio y en formación, sin eco en las decisiones gubernativas.

En otras palabras, la estrategia debe desplazar el centro de formación que está focalizado en preparar estudiantes para presentar pruebas de Estado, dirigiéndolo a la identificación de las competencias específicas que requiere el estudiante para atender sus problemas inmediatos. Tales procesos tendrán que hacerse con carácter experimental y se validarán en la medida en que los procesos sociales de esas comunidades conviertan la educación en centro de desarrollo social, científico y económico, por tanto requiere procesos de formación para la participación de la comunidad y deconstrucción de los roles tradicionales de la sociedad, de la familia y de la escuela en la formación de niños, niñas y jóvenes, trabajando alrededor de dis- 
tintos centros de interés acorde con la necesidad, que en nuestro contexto sociocultural focaliza el centro de interés en el estudiante, en otros casos en los padres de familia y, en otros en las organizaciones comunitarias. Cada uno de ellos se convertirá en centro de atención y de formación prioritario en distintos momentos del proceso social.

Significa lo anterior que si bien es cierto que el proceso educativo debe centrarse en la formación integral del estudiante, hay que involucrar a padres de familia y a otros agentes educativos para que aprendan a participar, investigar, proponer y construir alternativas, siendo la meta el desarrollo social de su comunidad, meta que demanda convertir a la escuela en eje de ese desarrollo comunitario.

En ese orden de ideas, las políticas nacionales, regionales y locales servirán de punto de referencia, de criterios de orientación y de apoyo logístico, porque los Proyectos Educativos Institucionales (PEI) y los currículos para desarrollarlos estarán basados en las necesidades detectadas en los diagnósticos de la comunidad específica.

En últimas, se trata de invertir la mirada, transformarla y ampliarla proponiéndose partir de procesos micro con apoyo y referencia de los macro y no a la inversa, como se hace actualmente, a fin de buscar en consecuencia impactos locales en los procesos de las comunidades específicas, independiente de los efectos nacionales e internacionales.
Seguiríamos "botando corriente" y echando la plata en un barril sin fondo si continuamos enajenados en la a-criticidad social frente a los despropósitos de las políticas educativas Estado-céntricas, puesto que como se ha demostrado no conducen a ningún lado.

No de otra manera se gestarían las condiciones para una movilización social por una sociedad fundada en los conocimientos, condición sine qua non para la transformación de las prácticas administrativas educativas, de camino al logro del ideal de ciudadano y sociedad participativa y con valores democráticos, competente para dinamizar la endogenización de su propio desarrollo humano, social y productivo, aun en un contexto como el nuestro, signado por la mundialización de la economía de mercado, en los términos establecidos por el interés privado.

Al caracterizar el problema de la des-administración pública de la garantía del derecho social fundamental a una educación de calidad como un problema de moralidad pública, la sociedad colombiana puede concienciar el problema en términos de legitimidad política del Estado educador colombiano e incapacidad administrativa del modelo de la gobernabilidad para transformar las prácticas morales, políticas y educativas de sus operadores, y disponer su voluntad colectiva a controlar la ineficiente administración autónoma de la hipotética validez jurídica de la normatividad educativa de las competencias, colocando la administración pública del derecho social fundamental a una educación de calidad en términos de eficacia social, le- 
gitimidad política del Estado educador demandante de un cambio de estrategia, puesto que es evidente que no existe el interés ni la voluntad política de diseñar políticas de desarrollo social estructuradas, coherentes, en nuestro estudio de caso, con la visión de presente y futuro que dispusiera la sociedad y comunidad educativa en el Primer Plan Decenal Municipal de Educación.

Esta reconstrucción del funcionamiento sistémico del servicio educativo como proceso demandante de la construcción de indicadores de logros en los desempeños de las competencias administrativistas de los niveles político y técnico, desde los cuales evaluar la capacidad institucional universitaria y escolar a fin de encaminarla a objetivar el modelo de emprendimiento pedagógico emplazado en la política nacional de estándares y lineamientos curriculares por competencias, allanando por esta vía la carencia de articulación del Plan Sectorial de Educación con la jerarquía de objetivos estructurada en el Plan Decenal Municipal de Desarrollo Educativo de Pereira 2007-2016, es indicativo del camino para desarrollar el mandamiento de la formación integral en los contextos de vida de los educandos.

Finalmente, se debe empoderar una movilización social por la apropiación social de los conocimientos científicos y saberes ancestrales a través de la formulación participativa de una política pública de investigación, educación e innovación descentralizada, desde la cual orientar la acción del Estado en el nivel territorial para la profundización de la democracia participativa y la modernización del sector productivo, en el entendido de que la educación, al ser la puerta de entrada a la interculturalidad nacional y cosmopolita, no solo posibilita el desarrollo cognoscitivo para una participación ciudadana y una modernización del aparato productivo fundada en los conocimientos, sino también la formación del sentido eminentemente ético de una razón pública orientada por los valores y principios democráticos constitutivos del núcleo de constitucionalidad, tesis que hizo necesario establecer la relación entre los indicadores intermedios o de producto con los indicadores de impacto social o de resultado, relacionamiento encubierto por la unilateralidad de los indicadores de producto determinados desde un absolutismo administrativo Estado-céntrico que, al no medir los impactos sociales, elude la evaluación de la idoneidad de los desempeños administrativistas autonómicos territoriales e institucionales.

\section{REFERENCIAS BIBLIOGRÁFICAS}

\section{Referente normativo}

Constitución Política de Colombia de 1991. Bogotá: Edit. Temis.

Concejo Municipal de Pereira. Acuerdo № 56 de 2010. Plan de Desarrollo Municipal "Pereira Región de Oportunidades" 2008-2011.

Ministerio de Educación Nacional:

- Ley 30 de 1992.

- Ley 115 de 1994.

- Decreto 709 de 1996. 
- Decreto 2230 de 2003.

- Decreto 2832 de 2005.

- Decreto 409 de 2008.

- Decreto 4790 de 2008.

- Resolución 1036 de 2004.

- Segundo Plan Decenal Nacional de Educación 2006-2015.

Secretaría de Educación Municipal de Pereira (2007). Primer Plan Decenal Municipal de Educación 2007-2016. Pereira: Gobernación de Risaralda.

\section{Referente jurisprudencial}

Corte Constitucional:

- Sentencia T-002 de 1992.

- Sentencia T-418 de 1992.

- Sentencia T-419 de 1992.

- Sentencia T-571 de 1992.

- Sentencia SU-277 de 1993.

- Sentencia T-309 de 1993.

- Sentencia C-530 de 1993.

- Sentencia T-092 de 1994.

- Sentencia T-236 de 1994.

- Sentencia C-023 de 1995.

- Sentencia C-225 de 1995.

- Sentencia T-337 de 1995.

- Sentencia T-441 de 1995.

- Sentencia T-467 de 1995.

- Sentencia T-573 de 1995.

- Sentencia C-037 de 1996.

- Sentencia T-543 de 1997.

- Sentencia SU-225 de 1998.

- Sentencia T-019 de 1999.

- Sentencia C-578 de 1999.

- Sentencia T-881 de 2000.

- Sentencia T-944 de 2000.

- Sentencia T-1290 de 2000.

\section{Referente doctrinal}

Alexy, R. (2002). Teoría de los Derechos Fundamentales. Madrid: Centro de Estudios Políticos y Constitucionales.

Aristóteles (2007). Ética a Nicómaco. Madrid: Gredos.

Botero Uribe, D. (1999). Teoría Social del Derecho. Bogotá: Universidad Nacional de Colombia.

De Zubiría, J. (2011). "Resultados de la evaluación docente". En: Periódico El Tiempo.

Ferrajoli, L. (2009). Derecho y razón. Teoría del garantismo penal. Madrid: Ed. Trotta.

Freire, P. (2005). Pedagogía del oprimido. Buenos Aires: Siglo XXI.

Habermas, J. (2005). Facticidad y validez. Madrid: Ed. Trotta.

Hood, C. y Jackson, M. (1997). La argumentación administrativa. México: Fondo de Cultura Económica.

Hoyos Vásquez, G. (2005). Elementos para una teoría discursiva de la pedagogía a partir del pensamiento de Jürgen Habermas. Bogotá: Pontificia Universidad Javeriana.

Hoyos Vásquez, G. (2007). Borradores para una filosofía de la educación. Bogotá: Siglo del Hombre Editores. 
Jaeger, W. (1992). Paideia. México: Fondo de Cultura Económica.

Mejía Quintana, O. (2005). Teoría política, democracia radical y filosofía del Derecho. Bogotá: Edit. Temis.

MEN (1997). Lineamientos curriculares, educación ética y valores humanos. Bogotá: Magisterio.

Nussbaum, M. (2010). ¿'Por qué la democracia necesita de las humanidades? Madrid: Katz Editores.

Orozco Restrepo, G. y Gamba, G. (2010). Diagnóstico del Proyecto Sesquicentenario de Pereira. Pereira: Presentación Power Point.

Plan de Desarrollo Municipal de Pereira (2008-2011). Región de Oportunidades. Pereira.
Posner, G. (1999). Análisis del currículo. Bogotá: Ed. McGraw-Hill.

Presidencia de la República (1993-1994). Misión de ciencia, educación y desarrollo. Colombia al filo de la oportunidad. Bogotá: Tercer Mundo.

Prospectiva de ciudad de Pereira (2010). Análisis situacional. Documento digital. Tomado del Diagnóstico del Proyecto Sesquicentenario de Pereira.

Sabine, G. (2006). Historia de la teoría política. México: Fondo de Cultura Económica.

Searle, J. (1997). La construcción de la realidad social. Barcelona: Paidós. 